\title{
A NetLogo Implementation of the Norms and Metanorms Game: Behavior Analysis Meets Agent-Based Modeling
}

\author{
Diogo Conque Seco Ferreira ${ }^{1} \quad$ Julian Tejada Gehazi Ramiris dos \\ Santos Bispo
}
Departamento de Psicologia, Departamento de Psicologia, Secretaria Municipal Universidade Federal de Universidade Federal de Sergipe Sergipe de Saúde, Prefeitura
Municipal de Petrolina

\begin{abstract}
This work proposes filling the gap between cultural behavior analysis and recent computational approaches to the study of social dynamics. We first introduce a game-theory infused understanding of norms and metanorms evolution and how agent-based models are used to simulate and analyze the emergency of normative phenomena. Then we argue that this method is in line with the cultural behavior analysis approach to the evolution of culture, offering a common framework for further theoretical and heuristic developments for researchers in the field. We proceed by outlining Axelrod's Metanorms Model, followed by a tutorial on its implementation with NetLogo programming language. Finally, two experiments are carried out using this framework, and the implications of the results, along with future direction of research, are discussed from a Skinnerian perspective.
\end{abstract}

Keywords: agent-based modeling, norms, metanorms, social simulation, behavior analysis

Societies are able to prosper, in part, because individuals are often willing to help others even when it incurs costs for themselves (Rand \& Nowak, 2013). The existence of prosocial behavior challenges the basic game-theoretic assumption that humans are narrowly concerned with self-interest (Binmore, 1998). Prosocial behavior may be supported, in part, through third-party sanctioning, acts of approval or condemnation provided by an uninterested audience. When a transgression occurs, sanctioning behavior is more effective when it is administered by a third-party, as the victims of violations often lack the resources to retaliate or are incapacitated by the norm violation (Fehr \& Fischbacher, 2004). To demonstrate the pervasiveness of thirdparty sanctioning, Fehr and Fischbacher

1 Correspondence concerning this paper should be addressed to Diogo Conque Seco Ferreira, Department of Psychology, Federal University of Sergipe. Email: diogoseco@ufs.br 
(2004) allowed participants to act on the result of a Dictator Game by spending their own resources to decrease the dictators' earnings. Indeed, many participants opted to punish selfish dictators. Similar results have been observed using different procedures and various types of social dilemmas (Fehr \& Gächter, 2002; Henrich et al., 2006).

Examples of sanctioning behavior raise the question of why third-parties sacrifice resources to influence the outcomes of other parties. Sanctioning may induce prosocial behavior, but what motivates third-parties to engage in costly acts of sanctioning, be it rewards or punishments? Sanctions can be interpreted as a second-level public good (Yamagishi, 1986) because they benefit the collective interest by encouraging prosociality, a group level benefit, while bearing a cost to the individual sanctioners (Horne, 2007). Some have noted the danger of infinite regress in this reasoning (e.g., Kiyonari \& Barclay, 2008), as any prosocial behavior would require a higher-level explanation, making even the most trivial altruism act theoretically intractable (but see Horne, 2008; Sober et al., 1999 for opposing arguments).

Building on this understanding, Horne and Mollborn (2020) proposed a Relational Theory of Norm Enforcement, in which the social relations between members of a group play a fundamental role. Instead of exclusively focusing on personal benefits and costs of her behavior, a group member also cares about maintaining her social relations. Most of the tasks performed by adults in modern society presuppose the presence or help of others, and, in this way, maintaining an active and favorable social network is of the most importance (Guerin, 2003). Apart from the direct benefits of their behavior, members of a group have a collective interest in controlling each other's behaviors, rewarding good deeds and punishing wrongdoings. In this way, the sanctioning of metanorms (i.e., a second order norm enforcement) may be supported by mechanisms of costly signaling (Hardy \& van Vugt, 2006), in which the sanctioner acts to broadcast a favorable reputation for future interactions. Any costs of the metanorm sanctions will be canceled out by the increased quality of future interactions.

One important aspect of this model is that there are at least two patterns of behavior happening in the group: one in which the agents have to decide to contribute or not to a public good or a NPlayers Prisoner Dilemma, and another where the agents choose whether to sanction the non-cooperators. While the former may be related to the executive decisions agents face (decisions that impact how much resources they will have), the latter is related to how the resources should be generated (norms), and what to do with those who do not apply the norm (metanorms). Axelrod (1986) synthesized this dilemma in a model in which the costs of third-party sanctions are collectively borne by participants in the group. The enforcement of norms on how to conform to norms (metanorm) may bring about the necessary conditions for the maintenance of social norm and, being the norm beneficial to the average group payoff, the metanorm enforcers would be selected for.

The author describes a game in which norms, over certain circumstances, can evolve and even settle down in a community (Axelrod, 1986). His model 
considers a set of few variables which control the costs and benefits that an individual would obtain if she defected, in an environment in which it is possible that someone else observed her defection, and decided to punish. In the game, the norms were represented as the mutual agreement not to defect, and when an individual decides to break them, besides getting a benefit he or she could be punished. The model also incorporates a cost that the observer must pay to punish the transgressor, as well as a hurt that infringes on other group members each time a member decides to defect. The relationship between the benefit, the punishment and the hurt values defines the establishment of the norm. The model also includes a second level of norm enforcement, the metanorm, in which a second player has to decide whether to punish those who did not punish the defector, and as in the norm enforcement, the metanorm enforcement has defined cost and punishment values.

The analysis advanced by the author differs in method from previous presented works. Whereas traditional research on the topic, conducted by psychologists and economists, focus on verbal theoretical advancement closely tied with empirical laboratory experiments (Smaldino, 2020), Axelrod's work borrows the Agent-based Modeling (ABM) method from the emerging field of Computational Social Science (Epstein, 2006). Presenting itself as a third complementary way of building theory (Ostrom, 1988), ABM aims at explaining intricate aggregate level phenomena by exploring lower level interactions between agents in computer simulated environments. Once a set of features is defined at the agent level, it becomes possible to observe how the interactions between them, each acting individually, shape group-level phenomena. Epstein (2006) points to three defining characteristics of the method: (1) focus on the individual (agent); (2) its simplicity; and (3) its close ties with experimental methods. When applied to Social Sciences, ABM states that group-level regularities may be studied by looking at the local level interactions of autonomous and heterogeneous agents, a "bottom-up" approach. The precise characteristics of the agent behavior depends greatly on the researcher's objective, and it may vary from simple one-job agents in a static environment (Axelrod, 1994) to a complex society of agents interacting in everchanging situations (Epstein et al., 2000). In the Social Sciences, however, ABM advises to model simple agents interacting first, as it may point to the minimal requirements necessary to the emergence of aggregate-level phenomena. Lastly, the proposers of ABM acknowledge the close relationship between ABM and traditional experimental/empirical methods, such that ABM may generate interesting and testable empirical ideas (or may be used to expand and explore previous empirical findings (e.g., Smaldino, 2019). For a full introduction to $\mathrm{ABM}$ and its contributions to Social Sciences, refer to Epstein and Axtell (1996).

\section{Behavior Analysis of Norms and Metanorms Evolution}

Although Behavior Analysis has provided no detailed account of norms and metanorms evolution, there seems to be a sizable overlap in interests between 
behavior analytic researchers and those handling the issue from different theoretical perspectives. In a seminal paper outlining the role of verbal behavior in cultural practices, Glenn (1989) borrows concepts from Harris' Cultural Materialism to emphasize different levels at which individual behavior may be vital to cultural practices. At the infrastructural level, verbal and non-verbal behaviors are directed at solving problems posed by the immediate context. According to the "principle of infrastructural determinism", the survival of a culture depends on the efficiency and transmissibility of the behavioral relations maintained at the infrastructural level. The structural level is where members in a group establish practices to control the infrastructural design, and Glenn defines it as "comprised of political and domestic practices that regulate relations among individuals in the system and that function to support infrastructural practices. Structural practices include those having to do with domestic division of labor, socialization and education, discipline, and sanctions" (p. 12). So the cultural structure could be thought of as a set of norms aimed at controlling the efficiency of the infrastructural level, assigning merit or blame to infrastructural performance, and, also, to establishing sanctions to the enforcement of those consequences. Finally, the superstructural level emerges from the enacting of the two previous levels and may be thought as culture in itself, a product of intense negotiation between different infrastructural and structural demands met by the group.
In a recent development, Couto (2019) proposes a model of selection of cultures that advances similar arguments. The author uses the concept of Interlocking Behavioral Contingencies (IBCs) to describe the relations between members of a group and their environment and states that within-group behavior selection would be best conceptualized as selection of cultures, as it involves different IBC's competing for survival and reproduction. He subsequently distinguishes between execution IBC (eIBC) and controlling IBC (cIBC), so that the first involves social relations directly associated with the production of group's outcome (Aggregate Product, in metacontingency parlance) and the second involves social practices associated with the control of these social relations, which, in turn, guarantee the production of the group outcome and, thus are indirectly controlled by it. The eIBCs closely resemble the Harris' infrastructural level, while the cIBCs seem to relate to the structural level. $\mathrm{Be}$ it as it may, the cIBC's and the structural level both point to the norms and metanorms enforced by a group.

Considering an Elementary School as an example, the relations between the administration, staff, teachers, and students comprise the infrastructure or eIBCs, as they involve the production of the intended group outcome: establishing a basic repertoire on the students. On top of that, there exists a second set of relations that establish who should do what, when and how, and, moreover, what to do when those actions are not performed. These relations would encompass the structural level or cIBCs, and they guarantee the proper functioning of this social system. Some of those rules are enshrined in codes of conduct (civil law, constitution, code of 
ethics, etc.) but a sizable portion is informally enforced: teachers will complain if they see a colleague shirking, students may snitch on a colleague for vandalizing school property, etc. These practices are not directly related to the production of education (this system's main goal), but they specify the circumstances for the eIBCs to be most effective. And to fully comprehend this group's dynamics, one should investigate not only the executive social practices, but their controlling social practices too, as one does not stand apart from the other.

The main goal of the present paper is to introduce a game-theory infused understanding of norms and metanorms selection and evolution with the use of computer simulated social interactions. It is the position of the authors that this understanding is in line with the Skinnerian approach to the evolution of cultural practices presented earlier and that this method allows further theoretical and heuristic developments for researchers in the field. The paper will proceed first by outlining Axelrod's Metanorms Model, followed by a tutorial on its implementation with NetLogo programming language. Finally, two experiments are carried out using this framework, and the implications of the results, along with future direction of research, are discussed from a Skinnerian perspective.

\section{Method}

\section{Axelrod's Metanorms Model}

When a conceptual model is translated into an agent-based model the first step is to identify the agents and its actions, and how these actions affect each agent. In his model, Axelrod (1986) distinguished between three sets of agents: an executor, called agent $i$, and two observers, agents $j$ and $k$. Each one has a role of actions: the executor $(i)$ has to choose between to defect or not, while the observers (each $j \neq i$ ) have to decide whether to punish or not the defector, or other observer who did not punish the defector (for each agent $k \neq j$ and $i$ ). All roles are randomly assigned but, once the executor is defined, the selected agent maintains his role during the execution decision opportunities, while the other roles are randomly assigned after each executor's or observer's decisions. These actions affect the agent itself and other agents by producing certain payoffs (Table 1). Every time a defection takes place, the defector gets a benefit payoff, and if that defection is punished by an observer the defector gets a punishment payoff, and the observer who enforced the norm pays an enforcement cost. Also, this defection produces harm to everyone (but the defector) defined by the hurt payoff.

The second step is to define the agents' and environment variables that will control the behaviors and interactions in the simulation. In the Axelrod's model the agents are instantiated with just two parameters, their boldness and their vengefulness. The boldness parameter $\left(B_{i}\right)$ determines the probability of defecting, while vengefulness $\left(V_{i}\right)$ determines the probability of norm and metanorm enforcement (i.e., punishment of defections and shirking). In the original model, these two parameters (also called the agent strategy) are defined by a probability ranging from $0 / 7$ to $7 / 7$, randomly selected 
and fixed until the end of a round. The defecting decision is moderated by an exogenous parameter called $S$, defined by a random number drawn from an uniform distribution ranging from 0 to $1 . S$ determines the probability of a defection being seen by the group, and whenever $S$ $<B_{i}$, the agent $i$ chooses to defect. $S$ also determines the probability of a given agent $j$ observing a defection and an agent $k$ observing a shirking. Whenever an agent $j$ or $k$ detects a defection or shirking (respectively) with the probability $S$, the decision to punish the defector or the shirker is determined by their probability $V_{i, j}$.

Table 1. Original payoffs values of the Axelrod (1986) model.

\begin{tabular}{ll}
\hline \multicolumn{1}{c}{ Behavior } & \multicolumn{1}{c}{ Value } \\
\hline Defect benefit & 3 for the defector \\
$\begin{array}{l}\text { Hurt of } \\
\text { defection }\end{array}$ & $\begin{array}{l}-1 \text { for all but the } \\
\text { defector }\end{array}$ \\
Punishment & -9 for the defector \\
Enforcement & -2 for those who \\
cost & punish \\
\hline
\end{tabular}

A third step consists in defining the simulation procedures in a logical, step by step, workflow. In the original Metanorms Model, a set of 20 agents first must decide to cooperate or defect, each at its turn (N-Person Prisoner's Dilemma game). If $\mathrm{s} /$ he decides to cooperate (i.e., not defect) the procedure continues and another agent is selected for defection. If $\mathrm{s} /$ he defects, then surrounding agents have to (1) decide to punish or not the defector (Norms game), and (2) decide to punish or not those who refrained from punishing the defector (Metanorms game). After each action takes place the payoffs are assigned to each agent. After every agent has four opportunities for defection, an evolutionary selection process begins and a new generation of agents is created. The agents are ranked by their absolute earnings and divided in three tiers: the agents with the best scores generate two offspring with identical parameters, the middle tier agents provide one offspring, while the least scoring agents just die out. At the end of the process, there is a mutation process added to the system by selecting at least one random agent and changing their parameters with a fixed probability of $1 \%$.

In this way, Axelrod's ABM provides conditions to test theoretical predictions in a closed system that allows iterated interactions in a population of agents behaving in accordance with a simple set of rules (Axelrod, 1986) and has helped to elucidate the elusive dynamics of norm enforcement observed in the empirical literature (see Horne, 2006). The parameters presented in Table 1 may be interpreted as the agents' contingencies of reinforcement, where each action (response) produces a payoff (consequence), while their boldness and vengefulness could be interpreted as their probability of emitting defective and punishing responses. As discussed earlier, we propose that the contingencies controlling the executor's behavior are eIBCs, while the contingencies for norm and metanorm enforcement comprise the cIBCs. The selection procedure, in the current interpretation, is seen as a process of evolution of the agents' behavior. Even though the terms generation and offspring are used, they can be 
understood as rules to update agents' probabilities of emitting either response: defection or punishment. At the end of a number of interactions (i.e., a generation), agents access which strategy was most successful and copy it. Mutation is added to include noise (such as errors in establishing the winning strategy or in implementing the best behavior) and variability (such as the appearance of a new member in the group) to the agents' behavior.

\section{NetLogo}

NetLogo is a development environment for multi-agent simulation that contains an extensive library of example models covering a wide variety of knowledge areas. It is a freely available, open-source, multi-platform software, with its own programming language of same name (Chiacchio et al., 2014; Wilensky, 1999). Its name is derived from the educational programming language Logo and it was designed for both teaching and research purposes, in special, for students or researchers without a programming background.

It provides a virtual environment in the form of a grid, called world, in which different types of agents can interact with each other. In NetLogo there are four types of agents: patches, turtles, links and the observer. Each one has a function and a set of features. Patches are fixed pieces of "ground" that make the world. Turtles can move through patches in any distance/direction at every step of time, and can assume different shapes and sizes. Links have the function of connecting at least two turtles, and are represented by lines. The observer is the overseeing agent that gives other agents instructions and makes changes in the world. It also owns a set of global variables that define general parameters of the model which can be accessed by the other agents. Also, each turtle, patch and link have their own set of variables and their own initial conditions, being able to inherit or transmit these properties, which offers the possibility of combining environmental, social and biological (evolutionary) dynamics in a single model.

The time passes as discrete steps called ticks, in which all agents can behave (one at a time), reducing significantly the lines of code, once at every tick all agents can be asked, for example, to move using only one line of code. NetLogo also offers an interface where the simulation can be controlled, variables can be manipulated and graphs with simulated data can be plotted. In this interface it is possible to observe the ticks count, the world and the agents behaving, as well as to control the flow of the simulation and variables values through buttons, switchers, sliders and other user interaction elements. As an alternative to this interactive type of simulation, NetLogo offers a built-in tool called BehaviorSpace that allows the user to perform simulation experiments with a large parameter space to test different model assumptions and generate simulated data for further analysis, based on a multicore parallel computation paradigm.

\section{Results}

\section{The NetLogo Model}


In our NetLogo implementation of the Axelrod's Metanorms Model we used only three agents: turtles, patches and the observer. From these, only turtles and the observer had function in the simulation. The turtles were the agents who behaved accordingly to the set of actions defined in the original model (described earlier), and the observer was the agent handling all instructions from the code to the turtles, as by default in any NetLogo simulation. The world consisted of a 16x16 grid made of patches where the turtles stood on, and at every tick one turtle had four opportunities to defect, as well as other turtles had the opportunity to punish the defector or punish a shirker. The main global variables were the number of agents (turtlecount), the $S$ parameter and the payoffs values. The $S$ parameter was implemented by the seen? variable defined by a random number ranging from 0 to 1 . The number of agents and the payoffs were set up to the Axelrod's model default values, but we also implemented sliders to manipulate these values. Finally, as by NetLogo default, we added setup and some Go buttons to initialize and control simulation flow, respectively.

\section{Definition of Turtles-Own Variables}

In the NetLogo context, a turtle has a set of variables called turtles-own variables which define their form, color, position and other properties. Some turtle variables like form, color, position and movement are built-in variables that can be manipulated, but others are custom. Most of the custom variables control a turtle actions, and for the case of Axelrod's model implementation, they controlled the decision of the turtles. As in the Axelrod's model, turtle agents have to decide whether or not to defect, so it is necessary that each agent has a variable that defines its tendency to defect (boldness). Similarly, an agent must have a variable that defines its tendency to punish (vengefulness), and finally, a variable that stores the payoffs produced after each decision (score). Figure 1 represents the initial condition in which the agents and their variables are created. Different from the original model, the boldness and vengefulness variables are initialized with random numbers from the interval $[0,1]$. The variable score is initialized as zero.

\section{Simulation Procedures}

The simulation algorithm is depicted in Figure 2. Once the environment has been initialized (time 0), an agent $i$ is randomly selected to choose whether or not to defect by comparing its boldness $\left(B_{i}\right)$ versus a generated random number $S$ (seen? variable represented by a flipped coin). If its boldness is greater than $S$, then the agent defects, otherwise it does not defect. Once an agent defects, the hurt payoff $(-1)$ is assigned for each other agent and the defection benefit payoff (3) is assigned to the defector. After that, the Norms game starts with another agent $(j)$ randomly selected to choose whether or not to punish the defector. First is evaluated if agent $j$ saw agent $i$ defecting with the probability $S$. If $j$ observes $i, j$ has to decide whether or not to punish $i$ with the probability $V_{i}$. If $j$ decides to punish $i$, the enforcement cost and punishment payoffs are calculated for $j$ and $i$, respectively. Finally, when an agent ignores a defection the Metanorms game 
starts by randomly selecting another agent $(k)$ to choose whether or not to punish who ignored the defection, and a similar procedure to the Norm game occurs. A run stops when all agents had the opportunity to defect four times, after that, the total scores are computed and the best agents will have the opportunity to transmit their own variable values to offspring, following a genetic algorithm process.

The genetic algorithm is an evolutionary dynamic procedure in which the best agents (in terms of their scores) will be selected to compose the next generation, including not only the selection of who has the opportunity to transmit their "genetic" information but also the way in which this information will be passed to the offspring (reproduction). Axelrod (1986) proposed that it could be interpreted as an asexual reproduction, because the agents generate offspring with identical parameters, but the process of transmitting "genetic" information could be improved if, in addition to asexual reproduction, procedures which emulate sexual reproduction were included. These procedures are called crossover reproduction, and they consist of mixing the parameters of the two agents to generate an offspring.

Here the two types of reproduction were implemented, along with mutation, and a new generation was composed of a set of the best agents which remain after the generation, a set of agents created by the crossover of the best, and a group of totally new agents. The process of mutation is done by randomly selecting an agent (with a probability of 0.01 ) of the new generation and changing one of their parameters. In our case, the best five agents remain in the population, and their values of boldness and vengefulness are mixed to create new agents following the crossover procedure. For this, two agents of the best five are randomly selected to produce two new agents, one of them will have the boldness of the first selected agent and the vengefulness of the other, and so on. After that, the new generation will be formed by the best five agents of the last generation, ten agents from the crossover, and five completely new agents.

Two experiments were performed through the BehaviorSpace tool. The first one aimed to replicate the results of Axelrod (1986) which evaluated the effects of a metanorms procedure on the establishment of norms. The second one evaluated the effects of changes in the Enforcement cost and in the $S$ parameters over total defections. All agents and variables were set up by the original model defaults, except when cost was manipulated in Experiment 2. The experiments were carried out in NetLogo version 6.1.1. For those interested, the source code of the model implementation is available on GitHub at the following address: https://github.com/julian-tejada/Metanorms game/.

\section{Model Implementation and Experiment Results}

The interface of the NetLogo Metanorms Model implementation is presented in Figure 3 where the different elements for controlling the model parameters are specified. The Go button runs the simulation for only one agent, the go20 for one generation of agents, and the go*100, runs 100 generations of agents. All 
other elements (the switcher and sliders) allow changing the number of agents and generation duration, as well as the values of the defect benefit, hurt of defection, enforcement cost and punishment. Also, different charts were set up to follow the dynamics of agent's behaviors across the simulations.

Regarding the first experiment, the NetLogo implementation of the Metanorms Model reproduced the original model (Axelrod, 1986) results and the results of other replications (Matthews, 2016; Prietula \& Conway, 2009), by showing similar patterns for both boldness and vengefulness in the Norms and Metanorms games. After 100 generations run, the average boldness values were higher than vengefulness values in the Norms game (see Fig. 4A and Fig. 4B), while in the Metanorms game these patterns were reversed (Fig. $4 \mathrm{C}$ and Fig. 4D). The same pattern was observed for the number of defections (see Fig. 5), indicating that metanorm enforcement suppresses defections by significantly reducing average boldness in the population over repeated iterations. This effect is more prominent after long simulation runs. Results from 1000 generations run exhibits a similar pattern found by Prietula and Conway (2009) evidencing a reduction in the average of boldness and a high increase in the average of vengefulness in the Metanorms game (see Fig 6).

Regarding the second experiment, the Enforcement cost parameter varied from 0 to 9 and observed its effect on the average number of defections after 100 generations for each of the cost values (Figure 7). Increasing the costs of cIBCs over the value of 5 leads to a complete breakdown of cooperation, ceteris paribus. The effects on norms, however, are much less drastic, with desertion rate stabilizing over the value of 3 . This suggests that Metanorms are much more sensible to their cost of enforcement than Norms.

The other parameter variation was on the range of values of the seen? variable ( $S$ in Figure 2). Values varied from 0.25 to 5 , and observed its effects over the average number of defections after 100 generations run for each value (Figure 8). Higher values indicate that the transgressions are more visible, hence, they have higher lower probability of being emitted (only agents with high values of boldness will defect) and higher probability of being punished (more surrounding agents will observe the transgressions). Our results indicate that metanorms are most needed for seldom seen transgressions, where they maintain defections at clearly lower levels than with norms alone. The easier to observe the transgression, the less practical relevance a metanorm has.

\section{Discussion}

Our goals in this paper were to develop a NetLogo implementation of the Norms and Metanorms game (Axelrod 1986) and to propose an interpretation from a Skinnerian perspective of cultural evolution, as indicated in the Introduction. The discussion will be divided in three parts. First, the technical details of the model will be discussed. Then, the uses and benefits of model building for Behavior Analysis is briefly considered, before the parallels between the present model and the Skinnerian account on the evolution of cultural practices is examined closely. 
Due to the lack of formal clarification in the original model (Axelrod, 1986), the present implementation required adjustments in some arbitrary assumptions and procedures of the model. The main alterations were carried out in the range of values that the boldness and vengefulness variables could assume, and in the evolutionary procedure by updating the genetic algorithm, to include, besides mutation, a crossover reproduction. This made possible amplify the parameter space of the main agent variables and allowed parameter mixing in the new generations, giving the model more granularity and stability. Despite that, the new model fits well with the previous results of other replications (Prietula \& Conway, 2009), presenting similar dynamics on the vengefulness and boldness variables, as well as the number of defections for the Norms and Metanorms games. As the model source code is available on a GitHub repository, it is possible other researchers interested in this kind of implementation reproduce the main findings and make further extensions of the model. The available version permits a flexible configuration of different parameters and observation of their effects over the agents behavior.

Regarding the interpretation of the model, in the introduction of his book aptly called Generative Social Science: studies in agent-based computational modeling, Epstein (2006) states that to understand the emergence of macroscopic societal regularities, one must answer the question: "How could the decentralized local interactions of heterogeneous autonomous agents generate the given regularity?" (Epstein, 2006, p. 5)
In the model herein presented, the question could be restated as follows: "How could a decentralized group of heterogeneous agents interact with the environment and with themselves to generate either norm compliance or norm defiance, a group regularity?". This question can be addressed by different types of scientifically sound answers, and it is indeed the case. Psychology and Social Sciences in general, Behavior Analysis included, have traditionally answered this question by creating verbal theories, verbal descriptions of events, concepts and their entanglement (Smaldino, 2020). Despite the mastery in writing skills by some of Psychology's most prominent writers, ordinary language has limitations as to its vagueness and ambiguity. Even when a precise scientific language is adopted, most verbal accounts make it difficult to understand the premises and fine details of their predictions (Gilbert \& Terna, 2000). In the context of Evolutionary Biology, Servedio et. al. (2014) state that verbal models in his field lack the clarity and precision needed to account for the complexity of the phenomenon. The authors champion the use of models, in their case mathematical models, as a way of carrying out "Proof-of-Concept Models", models inspired by verbal theories but with explicit assumptions. In this way, these models can be used to judge the appropriateness of verbal theories, precisely describing results of the events and relationships they propose.

Consider this excerpt of verbal theory: "men act upon the world, and change it, and are changed in turn by the consequences of their action." (Skinner, 1957). It is the opening line of Skinner's most important work and it puts forth the basic tenet of Radical Behaviorism: 
behavior is the result of ongoing interactions of an organism with its environment. As enlightening and insightful an idea as it is, a verbal formulation of such complex phenomenon is bound to be incomplete, leaving the intricate details of the continuous dynamics untouched (Resnick, 1994). According to Ostrom (1988), there are two more "symbol systems" available to the social scientist: mathematical models and computational models. Mathematical models are well established in the Skinnerian community (e.g., Baum's Generalized Matching Law, 1974) and their goal is precisely to establish the relevant elements in a situation, as well as the nature of their relationships, and then to observe their interaction to establish a benchmark against which empirical data is compared to. Computational models, on the other hand, are extremely rare. McDowell and colleagues have developed an intriguing and fruitful research project simulating behavior selection in a computer environment (McDowell, 2013, 2017, McDowell \& Klapes, 2019). Their implementations have to make explicit the implicit assumptions adopted by verbal theories and in the process they are able to not only specify these parameters, but also show how the parameters interact when the programs run, and to determine the limits of the model, such as how much variation is needed for a selection-by-reinforcement to be adaptive (McDowell, 2017). Agentbased modeling has the same benefits, and an additional one of doing away with most algebraic skills necessary for mathematical model building.
In a series of papers discussing the role of models in Science and Behavior Analysis, Marr (1992, 1993, 1996, 2009) advocates a Dynamic Systems approach to behavioral studies, with a clear focus on descriptions of behavior change and the conditions bringing about that change. Dynamic Systems, such as social practices, are particularly difficult to be verbally described because (1) they engender different levels of organization (individual, group and structural/situational) and (2) different ways in which these levels interact, and also because (3) these systems have "memory", that is, past events determine the state of a system on any given time. All three challenges are accommodated by Agent-based Modeling, such as the one presented here (cf. Smaldino, Calanchini e Pickett, 2015). In the present model, individual behavioral propensities are formally defined, the interactions between agents are established and the structure of their environment is clearly determined. At the beginning of a run, it is impossible to predict exactly when and how the behavior of the population will tend to norm conformance just looking at one agent or even at the agents mean propensities, without considering their environment and how they interact.

In our model, with the right parameters we can observe the emergence of norm compliance in a scenario where agents are following simple specified rules for their action. The concept of emergence has received a lot of attention by researchers interested in the study of cultural evolution. Although it is beyond the scope of this paper to present a full account of the debate (see Krispin, 2006, for a critical position), suffice it to say that the emergent property of the system is fully accounted 
for by the specifications of the model. Nothing else needs to be added. The emergent property of our model is simply "arising from the local interaction of agents" (Epstein \& Axtell, 1996, p. 35). In behavior analytic terms, the norm compliance pattern observed in our model is the product of eIBCs together with cIBCs, and changing any parameter of each contingency, may have dramatic effects on the probability of compliance emergence: as shown in Figure 8, once the cost of sanctioning exceeds 5 , the rate of defections sharply increases.

It is important to underscore that notwithstanding our model being able to generate a macro level phenomenon, it does not make its basic assumptions necessarily true. ABM can provide just sufficient explanations, not necessary ones. In fact, any complex phenomenon composed of interplay between levels may be implemented in different ways on lower levels (a property called multirealizability, Sawyer, 2005). Epstein (2014) calls such models candidate models, and specifies that an empirical research agenda must be conducted "figuring out which of the microspecifications is most tenable empirically. In the context of social science, this may dictate that competing micro specifications with equal generative power be adjudicated experimentallyperhaps in the psychology lab [emphasis added]" (p. 43).

\section{Conclusion}

This brings us to our final comment: the complementary relationship between ABM and empirical research. Natural sciences and engineering have adopted computer simulations as methods for a long time (Zeigler, 1976) focusing on prediction, such as predicting the position of a space station based on simulations of its trajectory. On Social Sciences, however, the principal value of simulations seems to be on theory development (Gilbert \& Terna, 2000). As mentioned above, proof-of-concept models may elucidate the adequacy of a proposed theory, while simple models like the one here presented may shed light on relevant parameters for a phenomenon of interest to occur. The complexity of a model is closely linked to available knowledge in a field, and when entering an area full of controversy and conflicting results, as is the case of evolution of cultural practices, it is advisable to start with a simple model.

\section{References}

Axelrod, R. (1986). An Evolutionary Approach to Norms. The American Political Science Review, 80(4), 10951111. https://doi:10.2307/1960858

Axelrod, R. (1994). A model of the emergence of new political actors. In Artificial societies: The computer simulation of social life, edited by Gilbert, N. and Conte, R. London: University College Press.

Axelrod, R. (1997) The dissemination of culture - A model with local convergence and global polarization. Journal of Conflict Resolution, 41, 2, 203-226.

Baum W. M. (1974). On two types of deviation from the matching law: bias and undermatching. Journal of the experimental analysis of behavior, 22(1), 231-242. 
https://doi.org/10.1901/jeab.1974.

22-231.

Binmore, K. G. (1998). The evolution of fairness norms. Rationality and Society, 10(3), 275-301.

https://doi.org/10.1177/104346398 010003001

Chiacchio, F., Pennisi, M., Russo, G., Motta, S., \& Pappalardo, F. (2014). Agent-based modeling of the immune system: NetLogo, a promising framework. BioMed Research International, vol. 2014. https://doi.org/10.1155/2014/9071 71

Couto, K.C. (2019) Tutorial: Selection of Cultures and the Role of Recurrent Contingencies and Interlocking Behavioral Contingencies. Behavior and Social Issues, 28, 37-45. https://doi.org/ $10.1007 / \mathrm{s} 42822-019-0001-y$

Epstein, J. M. (2006). Generative Social Science: studies in Agent-Based Computational Modeling. Princeton University Press.

Epstein, J. M. (2014). Agent-Based Computational Models And Generative Social Science. Complexity, 4(5), 4-46. https://doi.org/10.1515/978140084 2872.4

Epstein, J. M., \& Axtell, R. (1996).

Growing artificial societies: Social science from the bottom up.

Brookings Institution Press; The MIT Press.

Epstein, J., Dean, J., Gumerman, G., \& Axtell, R. (2000). Understanding Anasazi Culture Change Through Agent-Based Modeling. In G. J. Gumerman, \& T. Kohler (Eds.), Dynamics in Human and Primate
Societies: Agent-Based Modeling of Social and Spatial Processes (pp. 179-206). Oxford University Press.

Fehr, E., \& Gächter, S. (2002). Altruistic punishment in humans. Nature, 415(6868), 137-140. https://doi.org/ $10.1038 / 415137 \mathrm{a}$

Fehr, Ernst, \& Fischbacher, U. (2004). Third-party punishment and social norms. Evolution and Human Behavior, 25(2), 63-87. https://doi.org/10.1016/S10905138(04)00005-4

Gilbert, N, \& Terna, P. (2000). How to Build and Use Agent-Based Models in Social Science. Mind \& Society, 1, $1,57-72$.

Glenn, S. S. (1989). Verbal Behavior and Cultural Practices. Behavior Analysis and Social Action, 7, 10-15. https://doi.org/10.1007/BF03406102

Guerin, B. (2003). Language use as social strategy: A review and an analytic framework for the social sciences. Review of General Psychology, 7(3), 251-298.

https://doi.org/10.1037/10892680.7.3.251

Hardy, C. L., \& Van Vugt, M. (2006). Nice Guys Finish First: The Competitive Altruism Hypothesis. Personality and Social Psychology Bulletin, 32(10), 1402-1413.

https://doi.org/10.1177/01461672062 91006

Henrich, J., McElreath, R., Barr, A., Ensminger, J., Barrett, C., Bolyanatz, A., Cardaroas, J. C., Gurven, M., Gwako, E., Henrich, N., Lesoronol, C., Marlowe, F., Tracer, D., \& Ziker, J. (2006). Costly punishment across human societies. Science, 312(5781), 1767-1770. 
https://doi.org/10.1126/science.11

27333

Horne, C. (2007). Explaining norm enforcement. Rationality and Society, 19(2), 139-170.

https://doi.org/10.1177/104346310 7077386

Horne, C. (2008). Norm enforcement in heterogeneous groups: Sanctioning by majorities and isolated minorities. Rationality and Society, 20(2), 147-172.

https://doi.org/10.1177/104346310 8089543

Horne, C., \& Mollborn, S. (2020). Norms: an Integrated Framework. Annual Review of Sociology, 46, $467-487$. https://doi.org/10.1146/annurevsoc-121919-054658

Kiyonari, T., \& Barclay, P. (2008). Cooperation in social dilemmas: Free riding may be thwarted by second-order reward rather than by punishment. Journal of Personality and Social Psychology, 95(4), 826-842.

https://doi.org/10.1037/a0011381

Krispin, J. V. (2016). What is the metacontingency? Deconstructing claims of emergence and culturallevel selection. Behavior and Social Issues, 25, 28-41.

https://doi.org/10.5210/bsi.v25i0.6 186

Marr, J (2009). The natural selection: behavior analysis as a natural science. European Journal of Behavior Analysis, 10(2), 103-118. https://doi:10.1080/15021149.2009 .11434313

Marr, J. (1996). The mingled yarn. The Behavior Analyst, 19, 19-33.
Marr, M. J. (1993). Contextualistic mechanism or mechanistic contextualism? The straw machine as tar baby. The Behavior Analyst, 16(1), 59-65.

Marr, M. J. (1992). Behavior Dynamics: One Perspective. Journal of the Experimental Analysis of Behavior, 57, 249-266.

https://doi:10.1901/jeab.1992.57-249

Matthews, P. (2016). Going meta: Norm formation and enactment on the stack exchange network. In

Proceedings of the 25th International Conference Companion on World Wide Web ( $W W W^{\prime} 16$ Companion). International World Wide Web Conferences Steering Committee, Republic and Canton of Geneva, CHE, 781-785.

https://doi.org/10.1145/2872518.289 0592

McDowell, J. J. (2013). Representations of complexity: How nature appears in our theories. The Behavior Analyst, 36(2), 345-359.

https://doi.org/10.1007/BF03392319

McDowell, J. J. (2017). The Effect of Reinforcement, and the Roles of Mutation Rate and Selection Pressure, in an Evolutionary Theory of Behavior Dynamics. The Behavior Analyst, 40(1), 75-82.

https://doi.org/10.1007/s40614-0170094-9

McDowell, J. J., \& Klapes, B. (2019). An implementation of punishment in the evolutionary theory of behavior dynamics. Journal of the Experimental Analysis of Behavior, 112(2), 128-143.

https://doi.org/10.1002/jeab.543 
Ostrom, T. M. (1988). Computer simulation: The third symbol system. Journal of Experimental

Social Psychology, 24, 5, 381-392. https://doi.org/10.1016/00221031(88)90027-3

Prietula, M. J., \& Conway, D. (2009). The evolution of metanorms: Quis custodiet ipsos custodes?

Computational and Mathematical Organization Theory, 15(3), 147168. https://doi.org/10.1007/s10588009-9056-4

Rand, D. G., \& Nowak, M. A. (2013). Human cooperation. Trends in Cognitive Sciences, 17(8), 413-425. https://doi.org/10.1016/j.tics.2013 .06 .003

Resnick, M. (1994). Turtles, Termites and Traffic Jams: Explorations in Massively Parallel Microworlds. Cambridge, MA: MIT Press.

Sawyer, R. K. (2005). Social Emergence:

Societies as Complex Systems. Cambridge, MA: Cambridge University Press. https://doi: 10.1017/CBO9780511734892

Servedio, M.R., Brandvain, Y., Dhole, S., Fitzpatrick, C.L., Goldberg, E.E., Stern, C.A., et al. (2014). Not Just a Theory-The Utility of Mathematical Models in Evolutionary Biology. PLoS Biol, 12(12):e1002017.

https://doi.org/10.1371/journal.pb io. 1002017
Skinner, B. F. (1957). Verbal behavior. Englewood Cliffs, NJ: Prentice-Hall. Smaldino, P. E. (2019). Social identity and cooperation in cultural evolution. Behavioural Processes, 161(December 2017), 108-116.

https://doi.org/10.1016/j.beproc.201 7.11 .015

Smaldino, P. E. (2020). How to Translate a Verbal Theory Into a Formal Model. Social Psychology, 51(4), 207-218. https://doi.org/10.1027/1864-9335/a 000425

Smaldino, P. E., Calanchini, J., \& Pickett, C. L. (2015). Theory development with agent-based models.

Organizational Psychology Review, 5(4), 300-317.

https://doi.org/10.1177/20413866145 46944

Sober, P. E., Wilson, P. D. S., \& Wilson, D. S. (1999). Unto Others: The Evolution and Psychology of Unselfish Behavior. Harvard University Press.

Wilensky, U. (1999). NetLogo (6.1.1) [Computer software]. Center for Connected Learning and ComputerBased Modeling, Northwestern University, Evanston, IL.

Yamagishi, T. (1986). The provision of a sanctioning system as a public good. Journal of Personality and Social Psychology, 51(1), 110-116. https:// doi.org/10.1037/0022-3514.51.1.110

Zeigler, B.P. (1985) Theory of modelling and simulation. New York: Wiley. 
Figure 1

Definition of the Turtles-Own Variables

\section{boldness}

vengefulness

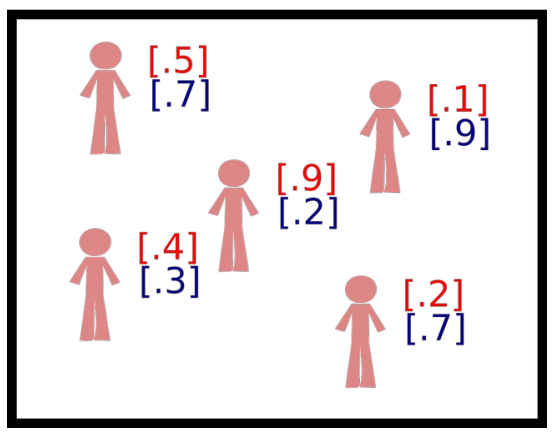

Note: Each turtle agent has two main variables, boldness or its tendency to defect, and vengefulness or its tendency to punish. 
Figure 2

Algorithm Structure of the Norms and Metanorms Game

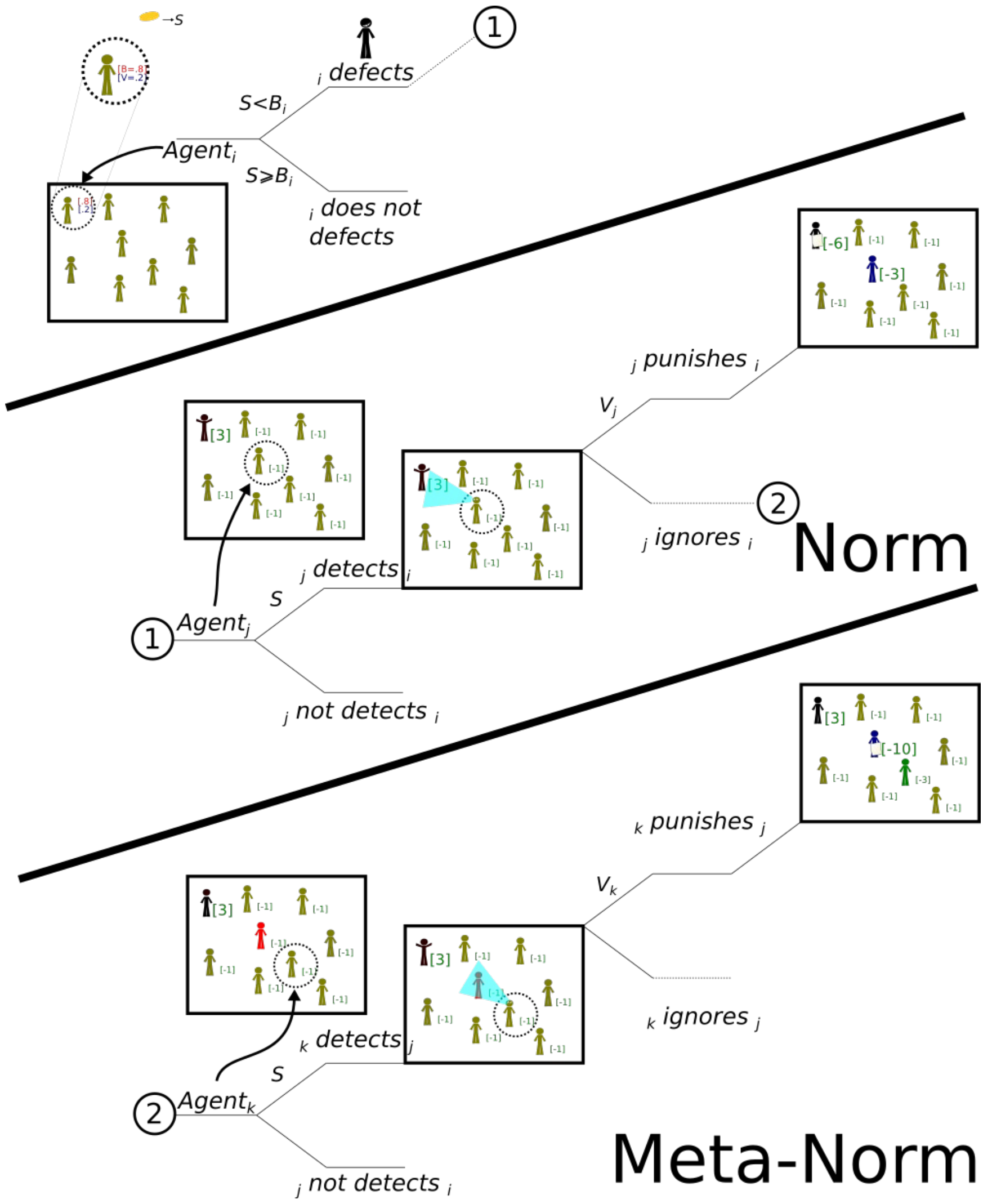


Note: $S$ represents the global variable seen?, letters $i, j$ and $k$ represent different agent sets and roles. Person-like shapes graphically represent turtle agents and adjacent numbers represent their scores. 


\section{Figure 3}

Interface of the Metanorms Model NetLogo Implementation

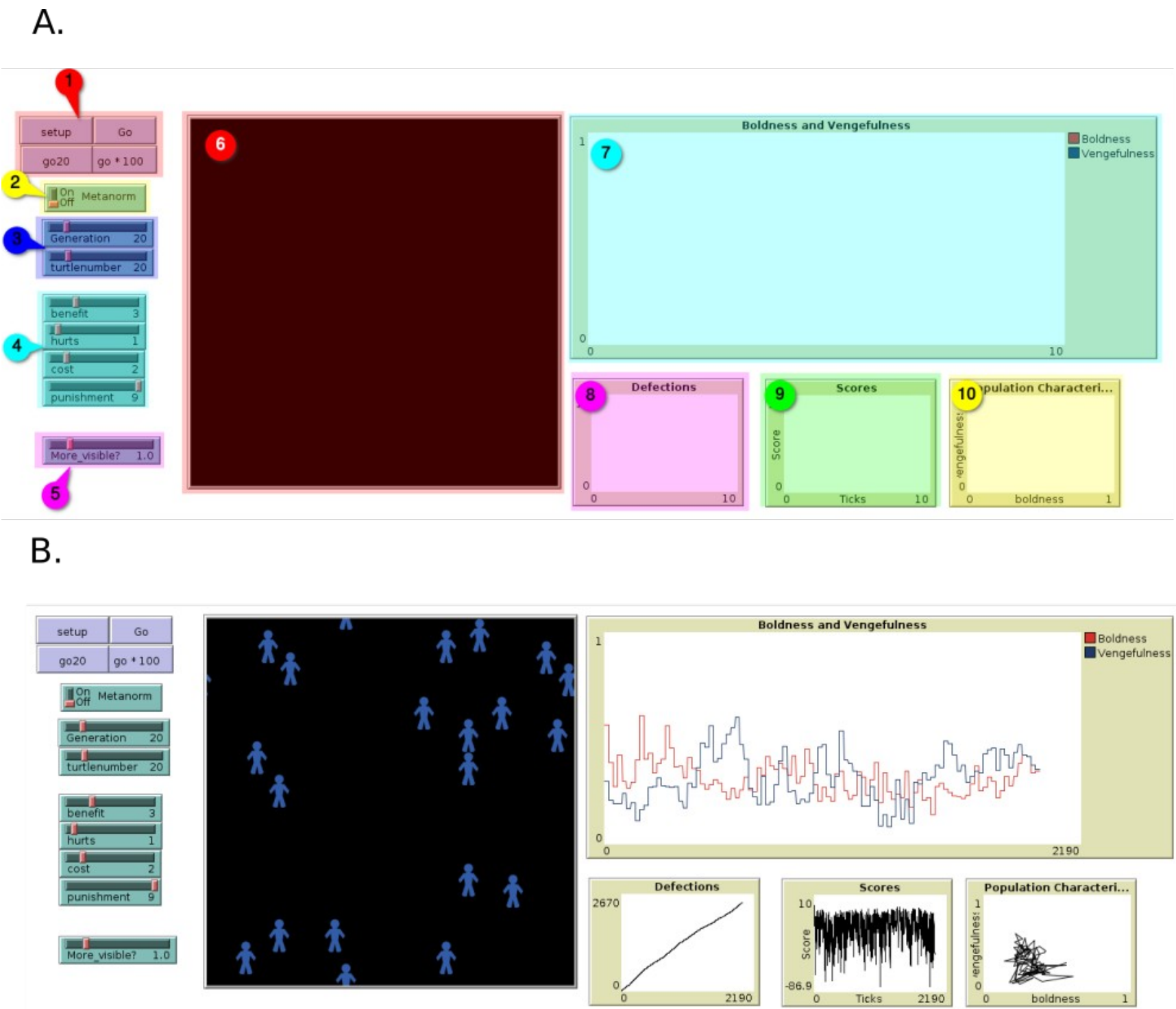

Note: In A, the NetLogo interface with the elements that control the simulation runs: 1. Control buttons to set up and run the simulation; 2. Switcher to turn Metanorms game on or off; 3. Slider to define the number of agents and the number of agents decisions which conform a generation; 5. Slider to define the value of the seen? variable; 6. World in which the environment and agents are instantiated; 7. Chart of the evolution of boldness and vengefulness variables across the generations; 8. Chart of the number of defections by generation; 9. Chart of scores by generation; and 10. Scatter plot of boldness versus vengefulness across generations. In B, the NetLogo Interface after 100 generations of the Norms game. 
Figure 4

Values of Boldness and Vengefulness by Game

A

Norms

$0.75-$

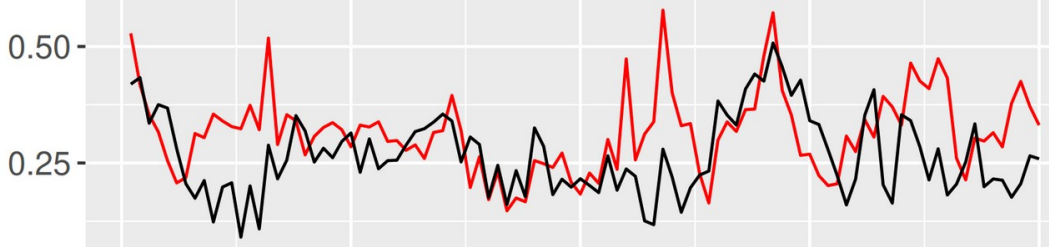

$0.00-$

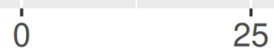

25

50

Gerations

c Meta-norms

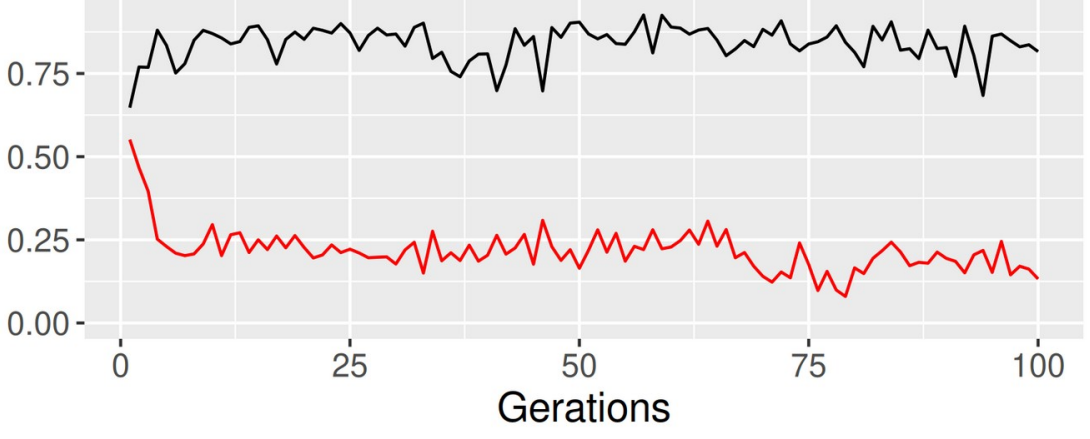

Groups — Boldness — Vengefulness
B Norms

0.75

0.50

0.25

0.00

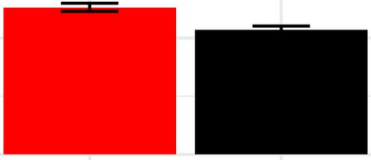

Boldness Vengefulness

D Meta-norms

$$
\begin{aligned}
& 0.75 \\
& 0.50 \\
& 0.25 \\
& 0.00
\end{aligned}
$$
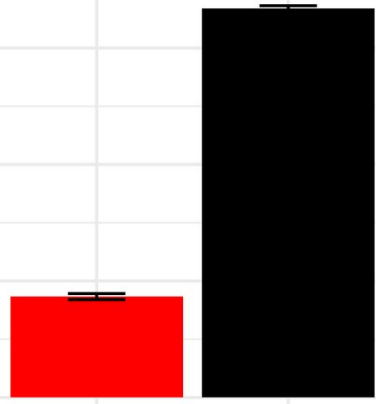

Boldness Vengefulness

Note: Evolution of boldness and vengefulness values across 100 generations for the norm (A) and metanorm (C) games. Average boldness and vengefulness values after 100 generations of the Norms (B) and Metanorms (D) games. 


\section{Figure 5}

Number of Defections by Game

A

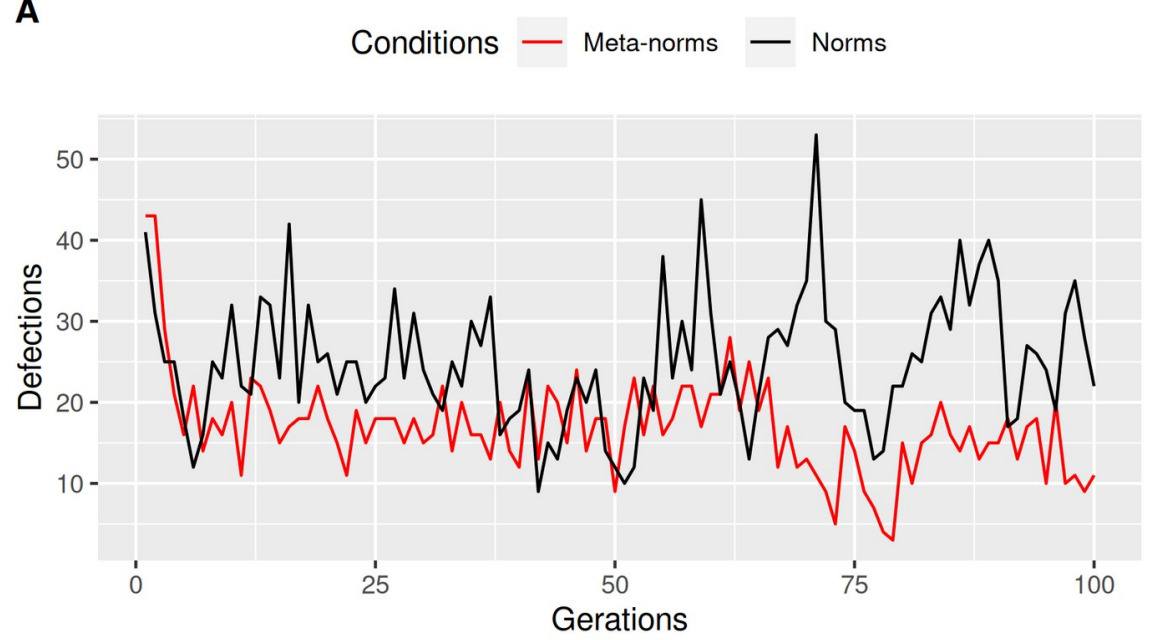

B

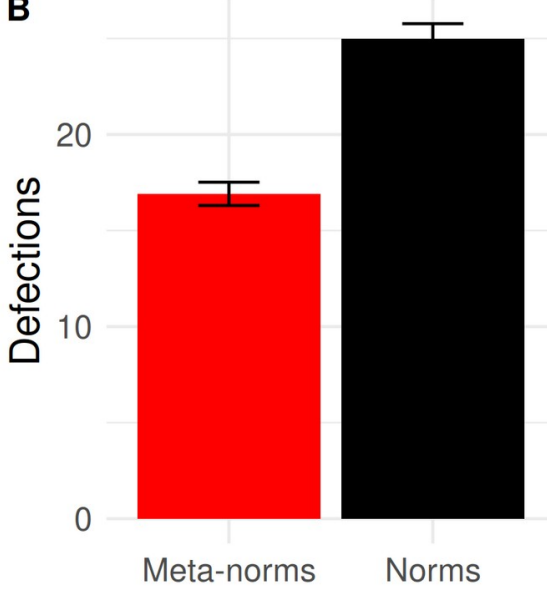

Note: (A) Evolution of the number of defections across 100 generations of the Norms and Metanorms games. (B) Average number of defections across 100 generations of the Norms and Metanorms games. 
Figure 6

Average Strategy Values by Game

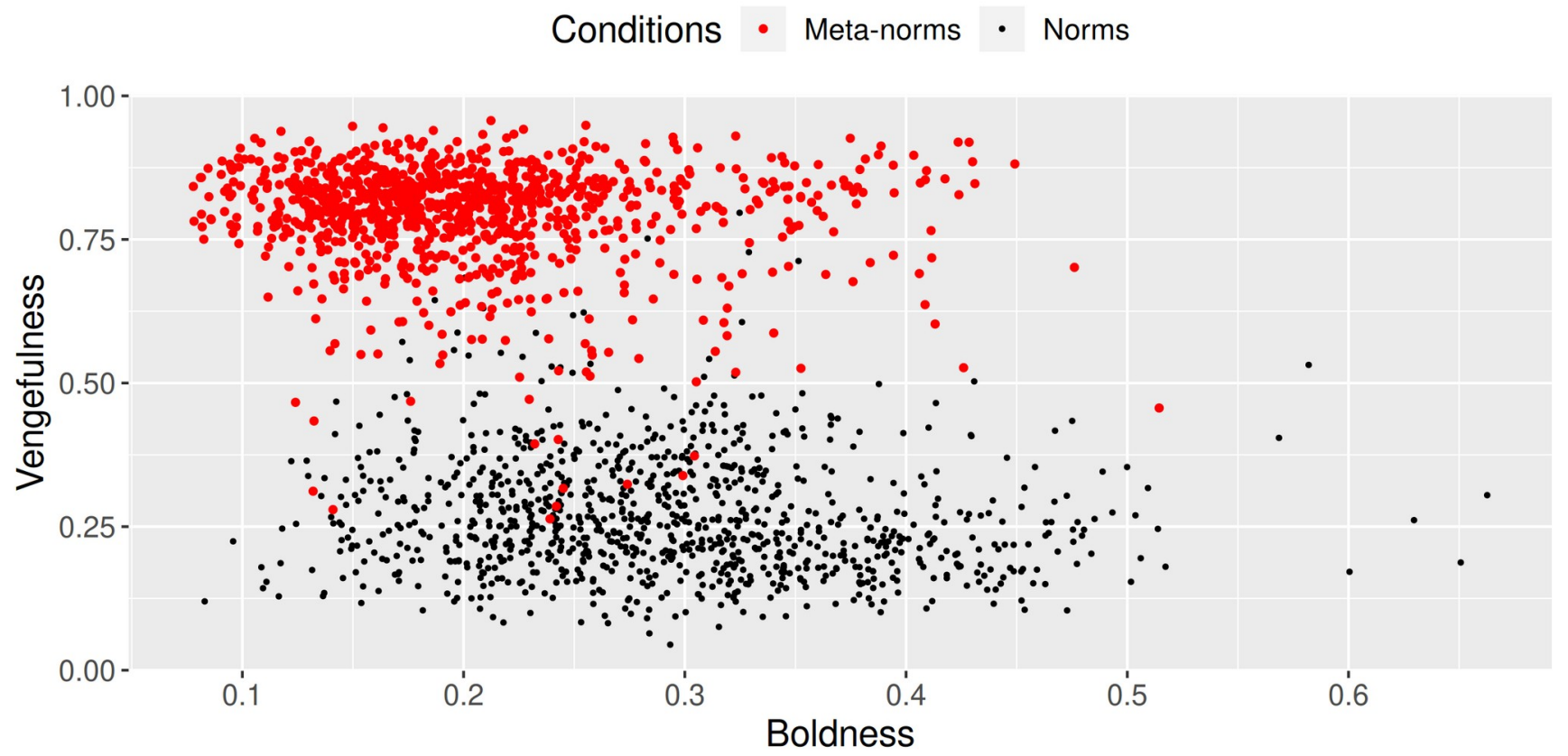

Note: Values plotted after 1000 generations. 


\section{Figure 7}

Average Number of Defections by Enforcement Cost Value in Norms and Metanorms Games

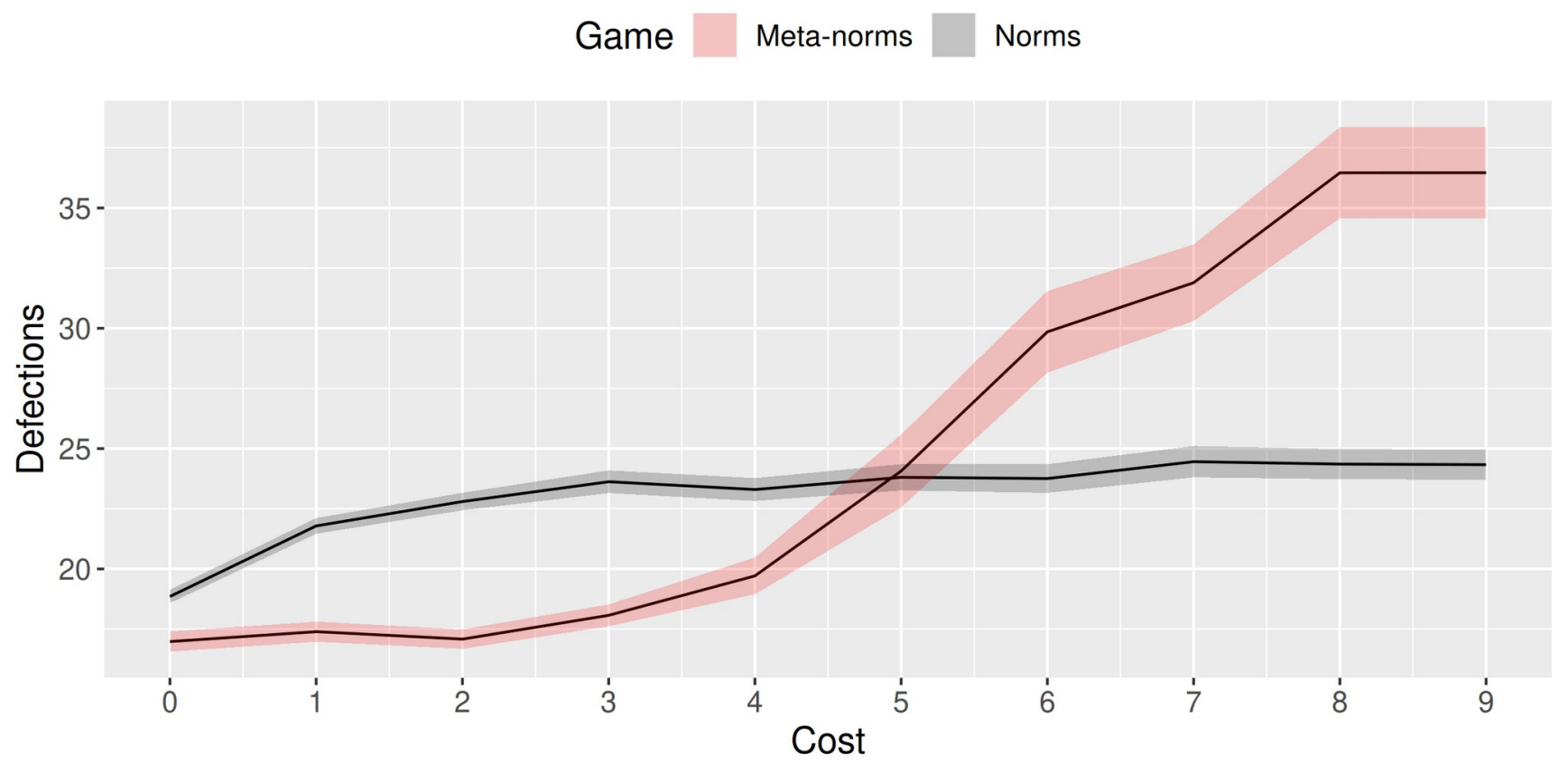

Note: Average number of defections for 100 generations in which the values of Enforcement cost were changed from 0 to 9 . The shadow represents standard error. 


\section{Figure 8}

Average Number of Defections by S Value in Norms and Metanorms Games

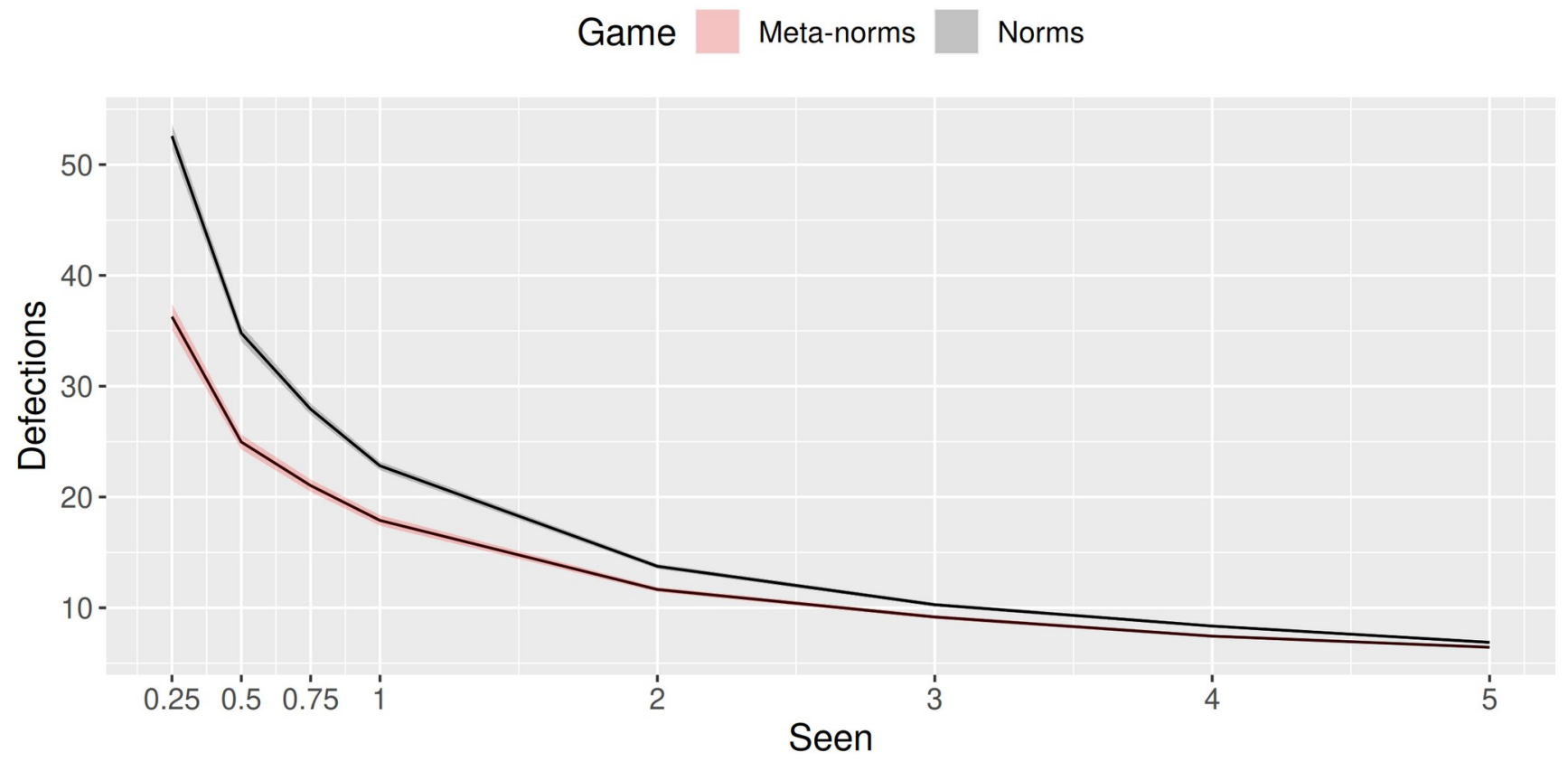

Note: Average number of defections for 100 generations in which the values of seen? $(S)$ variable were changed from 0.25 up to 5 . The shadow represents standard error. 